VOL. 16 (1977), 317-318.

\title{
Groups of automorphisms of linearly ordered sets: Corrigenda
}

\section{J.L. Hickman}

Dr Isidore Fleischer has shown by a very simple counterexample [1] that Theorem 9 ( $p .30)$ of my paper [3] is incorrect. The error occurs in the proof of Theorem 8 , and is due to my having interpreted certain group constructions as cartesian products, whereas they are in fact wreath products. The correct version of Theorem 8 is obtained by replacing cartesian products with wreath products in the appropriate places in Definition 5. The result, however, is easier to see than to state.

Let $\alpha$ be any ordinal, and let $\left(s_{\xi}\right)_{\xi<\alpha}$ be an $\alpha$-sequence such that each term $s_{\xi}$ is one of the following:

(1) an auxiliary symbol - either '(' or ')' ;

(2) a constant symbol - ' $Z$ ' (the infinite cyclic group);

(3) an operator symbol - either ' $x$ ' (cartesian product) or 'wr' (wreath product).

For the sake of brevity we assume as known or clear what we mean when we speak of such a sequence as being well-formed; that is, making grouptheoretic sense. We can now state the correct form of Theorem 8 as follows:

(a) A nontrivial group $G$ is a group of automorphisms of a scattered set if and only if $G \leq H$ for some group $H$ that is the interpretation (in the obvious sense) of some sequence of the type described above.

(b) A nontrivial group $K$ is a group of automorphisms of an ordered

Received 10 January 1977. The author wishes to express his thanks to Dr Fleischer and Professor Laver for drawing his attention to the errors here corrected. 
set if and only if $K$ is as in (a) or $K=G \mathrm{wr} H$ where $G$ is as in (a) and $H$ is a group of automorphisms of a nontrivial densely ordered set.

This result replaces Theorem 8 . Theorem 10 must be modified accordingly, and the word "dense" must be deleted from Theorem 11, yielding a result that is a direct consequence of the results by Holland and Neumann. Theorem 9 and its corollary must unfortunately be abandoned.

Professor Richard Laver has informed me that the result on pp. 14-15 that I attribute to Erdös and Hajnal is really due to Hausdorff [2], Satz XII, p. 458.

\section{References}

[1] Isidore Fleischer, "The automorphism group of a scattered set can be non-commutative", Buzl. Austral. Math. Soc. 16 (1977), 306.

[2] F. Hausdorff, "Grundzüge einer Theorie der geordneten Mengen", Math. Ann. 65 (1908), 435-505.

[3] J.L. Hickman, "Groups of automorphisms of linearly ordered sets", Buzz. Austral. Math. Soc. 15 (1976), 13-32.

Department of Mathematics, Institute of Advanced Studies, Australian National University, Canberra, ACT. 